\title{
Respiratory function, autonomic dysfunction, and systemic inflammation are closely linked in patients with COPD and tidal flow limitation: An exploratory study
}

\author{
Claudio Tantucci ${ }^{a}$, Damiano Bottone ${ }^{a}$, Guido Levi ${ }^{a}$, Silvia Uccelli ${ }^{\text {a }}$, Nicola Venturoli ${ }^{\text {a }}$, \\ Roberto Magri $^{\mathrm{a}}$, Emirena Garrafa ${ }^{\mathrm{b}}$, Laura Pini ${ }^{\mathrm{a}}{ }^{*}$ \\ ${ }^{\text {a }}$ Respiratory Medicine Unit, Department of Clinical and Experimental Sciences, University of Brescia, Brescia, Italy \\ ${ }^{\mathrm{b}}$ Department of Molecular and Translational Medicine, University of Brescia, Brescia, Italy
}

\section{A R T I C L E I N F O}

\section{Keywords:}

COPD

Autonomic dysfunction

Dynamic hyperinflation

Tidal expiratory flow limitation

Systemic inflammation

$\mathrm{C}$ reactive protein

\begin{abstract}
A B S T R A C T
Rationale: The study aimed to investigate the interplay among respiratory function, autonomic dysfunction, and systemic inflammation in COPD patients.

Methods: In 19 COPD patients, functional respiratory parameters, heart rate variability (HRV), and plasma highsensitivity-C-reactive-protein (hs-CRP) were assessed. Forced oscillation technique (FOT) was used to detect the absence (NFL) or presence (FL) of resting tidal expiratory flow limitation. Subsequently, patients underwent an incremental shuttle walking test (ISWT). Twenty healthy subjects were also shown as controls.

Results: $\mathrm{FEV}_{1}$, $\mathrm{DL}_{\mathrm{CO}}$, and lung volumes displayed significant correlations with $\mathrm{LH} / \mathrm{FH}$ ratio $\left(0.56<\mathrm{r}^{2}<0.27\right.$,p $<$ 0.01). A significant relationship was found between $\mathrm{LH} / \mathrm{FH}$ ratio with IC/TLC ratio $\%\left(\mathrm{r}^{2}=0.29, \mathrm{p}<0.05\right)$ and hsCRP $\left(\mathrm{r}^{2}=0.26, \mathrm{p}<0.05\right)$. Patients with FL had greater hs-CRP plasma levels $(\mathrm{p}<0.05)$, lower IC/TLC $\%(\mathrm{p}<$ 0.05), and higher $\mathrm{LH} / \mathrm{FH}$ ratio $(\mathrm{p}<0.001)$.

Conclusions: Worse airflow obstruction was associated with a higher LH/HF ratio, directly related, to hs-CRP and indices of dynamic hyperinflation. The presence of resting tidal FL with dynamic pulmonary hyperinflation is a strong driver of systemic inflammation and autonomic dysfunction.
\end{abstract}

\section{Introduction}

Autonomic dysfunction has been widely reported in patients suffering from chronic obstructive pulmonary disorder (COPD) (Mohamed et al., 2015). Abnormal modulation of autonomic nervous system (ASN) activity may occur in patients with COPD because of several complex mechanisms, such as altered chemoreflex (increased) and baroreflex (decreased) sensitivity, peculiar (rapid and shallow) breathing pattern, chronic pulmonary stretch receptors, and bronchopulmonary C-fibers stimulation, metaboreceptors involvement by inspiratory muscles load, hypoxic pulmonary hypertension, and therapeutic use of beta-2 adrenergic drugs (Mohamed et al., 2015; Van Gestel and Steier, 2010). In stable patients with COPD, there is an ASN activity imbalance with cardiac sympathetic predominance and disruption of autonomic reflexes (less ability to respond to sympathetic and parasympathetic stimuli) that can negatively affect cardiovascular and skeletal muscles function and influence the airway muscle tone and submucosal bronchial circulation, leading to impaired exercise tolerance and increased risk of cardiac morbidity and mortality (Heindle et al., 2001).

Finally, since the cholinergic system has been described as a modulator of the host inflammatory responses via cholinergic mediators acting on nicotinic acetylcholine receptors (nAChR) (Tracey, 2002; Wang et al., 2003), depressed vagal activity and, albeit less clearly, sympathetic dominance have been deemed capable of promoting inflammation in experimental models (Borovikova et al., 2000; Marz et al., 1998). As a logical consequence, in the attempt to explain the high frequency of multimorbidity found in this disorder, a relationship between autonomic dysfunction and systemic inflammation has been previously investigated in COPD, showing a significant association between IL-6 plasma levels and cardiac sympathetic dominance (Chhabra et al., 2015).

In this background, the study aimed to assess the interaction among lung function impairment, sympathovagal imbalance, and systemic

\footnotetext{
* Corresponding author.

E-mail address: laura.pini@unibs.it (L. Pini).
} 
inflammation in stable patients with COPD. A significant interplay of these negative factors might give reasonable pathogenetic support for developing systemic disorders typically occurring in this condition.

\section{Methods}

\subsection{Subjects}

This study was performed at the Respiratory Medicine Unit of the University of Brescia - Spedali Civili Hospital of Brescia, Italy, from November 2018 to May 2019. To be included in the study, subjects had to be aged 45-80 years and have had a diagnosis of COPD supported by the presence of risk factors, clinical judgment, and objective measurements of lung function according to ATS/ERS criteria $\left(\mathrm{FEV}_{1} / \mathrm{VC}^{2}<\right.$ LLN) (Miller et al., 2005). They had to be in stable conditions with no acute exacerbation in the previous 3 months, never or ex-smokers, and regularly treated with long-acting bronchodilators. The greatest possible attention was paid to exclude from the study patients with COPD suffering from notable chronic comorbidities by anamnestic self-reported medical history and medical records, if available (heart failure, ischemic cardiac disease, chronic atrial fibrillation, overlap with obstructive sleep apnea, chronic respiratory failure, Parkinson's disease and/or other neurological diseases, diabetes mellitus and/or other endocrine diseases) and assuming drugs such as beta-blockers, anti-arrhythmic drugs and any drugs capable of influencing the ANS activity. Twenty historical, age, and sex-matched, healthy subjects were also shown as controls for the heart rate variability (HRV) analysis from our lab.

\subsection{Study design}

In the morning, at rest and breathing room air, in seated position, each patient had an arterial blood gas sample for $\mathrm{pH}$, arterial gas partial pressures $\left(\mathrm{PaO}_{2}\right.$ and $\left.\mathrm{PaCO}_{2}\right)$, and plasma bicarbonates $\left(\mathrm{HCO}^{-}\right)$measurements. A venous blood sample $(5 \mathrm{~mL})$ was also withdrawn for high sensitivity $C$ reactive protein (hs-CRP) determination. The serum was separated, stored at $-80{ }^{\circ} \mathrm{C}$, and hs-CRP was measured later by an immune-nephelometric method (Dimension Vista 1500 - Siemens Diagnostics).

Subsequently, after an 8 -h wash-out from short-acting bronchodilators 48 -h wash-out from long-acting bronchodilators, each patient performed spirometry (BIOMEDIN Instruments, Padua, Italy) wearing a nose clip and breathing through a flanged mouthpiece. Slow vital capacity (SVC) and inspiratory capacity (IC) were measured twice using a bell spirometer at rest in sitting position. Then, at least three acceptable and reproducible maximal full expiratory maneuvers were performed to measure forced vital capacity (FVC), maximal expiratory volume in the first second $\left(\mathrm{FEV}_{1}\right)$, and maximal forced expiratory flows at different lung volumes.

Lung volumes were measured with a pressure-constant plethysmograph (BIOMEDIN Instruments, Padua, Italy). During the procedure, patients panted at a $0.7 \mathrm{~Hz}$ frequency. Three acceptable tracings of mouth pressure versus box volume changes were averaged to achieve a final measurement of functional residual capacity (FRC). Total lung capacity (TLC) and residual volume (RV) were computed subsequently. Then, by single breath technique, alveolar volume (VA) and coefficient of transfer factor for $\mathrm{CO}\left(\mathrm{K}_{\mathrm{CO}}\right)$ were measured twice to obtain lung diffusing capacity for $\mathrm{CO}\left(\mathrm{DL}_{\mathrm{CO}}\right)$, corrected for hemoglobin if needed (BIOMEDIN Instruments, Padua, Italy).

In each circumstance, the best values were retained for analysis. All tests were performed according to the ERS-ATS recommendations (Miller et al., 2005). Predicted values of lung function parameters were those proposed by the European Community for Coal and Steel (Quanjer et al., 1993).

Subsequently, by the forced oscillation technique (FOT), each patient had measurements during inspiration and expiration of total respiratory system resistance at different frequencies (R,rs5 and R,rs19) and respiratory system reactance at low frequency $(\mathrm{X}, \mathrm{rs} 5)$ with the determination of tidal expiratory flow limitation (FL), detected when the difference between expiratory and inspiratory reactance at low frequency $(\Delta \mathrm{X}, \mathrm{rs} 5)$ was greater than $2.81 \mathrm{cmH}_{2} \mathrm{O} / \mathrm{l} / \mathrm{s}$ (RESMON Pro Restech - Milan, Italy) (Dellacà et al., 2007).

In the late morning, a 5-minute HRV was recorded in each subject using a standardized procedure. In a quiet, comfortable room maintained around $22-26^{\circ} \mathrm{C}$, a standard lead II ECG was recorded in supine and then in orthostatic position in all subjects for twenty minutes. The analog signal was digitized using an A/D converter and stored on a computer with the data acquisition and analyzing software (Mat Lab V 6.1).

The recordings were manually examined, and regions with more than $1 \%$ ectopic beats or $5 \%$ artifacts were excluded from the analysis. The average heart rate of over 5 min was calculated. Time-domain and frequency-domain analyses using non-parametric Fast Fourier Transform (FTT) were performed following task force standards (Task Force of European Society of Cardiology and the North American Society of Pacing and Electrophysiology, 1996). In the frequency-domain analysis, the following were measured: Total Power (TP): the area under the spectral curve from 0 to $0.4 \mathrm{~Hz}$, a global index of HRV; VLF, power in the very-low-frequency band from 0.003 to 0.04 ; LF, power in the low-frequency band, from 0.04 to $0.15 \mathrm{~Hz}$, reflecting both sympathetic and parasympathetic activity; HF, power in the high-frequency band, from 0.15 to $0.4 \mathrm{~Hz}$, reflecting the parasympathetic activity; and low to high-frequency power (LF/HF) ratio averaged, reflecting sympathetic/parasympathetic balance. HVR parameters described above were obtained from a minimum of 3 artifact-free segments with average heart rate within $5 \%$ of each other, and the mean was retained for analysis. High values of LF and LF/HF ratio were considered to suggest increased cardiac sympathetic activity (Task Force of European Society of Cardiology and the North American Society of Pacing and Electrophysiology, 1996).

In the afternoon, $2 \mathrm{~h}$ after a light meal avoiding tea and/or coffee, each patient underwent an incremental shuttle walking test (ISWT). Briefly, after explanation and learning trials, each patient was required to walk repeatedly a $10 \mathrm{~m}$ distance, delimited by two road cones, at a progressively faster pace driven by the metronome sound. The ISWT was interrupted by the patient's inability to continue the exercise (breathlessness, leg muscles fatigue, or other symptoms) or to reach the cone at the right time. The number of meters was then calculated from the completed runs. By a pulse-oximeter (Nonin, Onyx Vantage), heart rate and oxy-hemoglobin saturation were obtained at baseline and continuously monitored throughout the exertion and also for the subsequent 5 min after stopping it in order to follow the heart rate recovery and calculate the heart rate decrease from the peak heart rate after $1 \mathrm{~min}$ $\left(\Delta \mathrm{HRR} t 1^{\prime}\right)$. A heart recovery rate of less than 14 beats in the first minute at rest was assumed to indicate cardiac autonomic dysfunction (Lacasse et al., 2005).

The study was approved by the local ethic committee, and all participants signed written informed consent upon enrolling.

\subsection{Statistics}

The variables of interest were analyzed by dividing the COPD patients into two subgroups according to tidal flow limitation (FL) and the absence of flow limitation (NFL). Unless specified otherwise, data are expressed as the mean \pm standard deviation.

Comparisons of different variables between Controls and all COPD and between two subgroups of COPD were assessed by the Student's unpaired $t$-test or the Mann-Whitney $U$ test if the normal distribution could not be assumed.

Correlations between variables were performed using Pearson's linear regression, and the coefficients of determination were computed. Statistical significance was accepted if $p \leq 0.05$. Statistical analyses 
were performed using Graph Pad Prism 6.0 (Graph Pad Software, La Jolla, CA) and SPSS 23.00 (IBM, Armonk, NY)

\section{Results}

The demographic characteristics of COPD patients and Controls (CTRL) are shown in Table 1.

Ten over 19 patients with COPD suffered from mild systemic arterial hypertension, 2 over 19 reported a previous episode of atrial fibrillation, and 5 over 19 had gastroesophageal reflux.

The patients in Table 1 and the following Tables are divided according to the absence (NFL) or presence (FL) of tidal expiratory flow limitation at rest in seated position. FL patients had significantly more chronic dyspnea (assessed by mMRC scale), a higher degree of BODE index, and were more hypoxemic and slightly hypercapnic at rest than the NFL patients (all $\mathrm{p}<0.05$ ). Two patients with previous atrial fibrillation and 4 patients with mild arterial hypertension and 4 with a history of gastroesophageal reflux were in the FL group.

The respiratory function parameters are reported in Table 2. $\mathrm{FEV}_{1}$ was $51 \pm 20 \%$ pred. in the whole group. Encompassing a wide range of airflow obstruction severity; mean lung volumes, i.e., RV, FRC, and TLC, were abnormally high but with a large dispersion, and a large standard deviation was also observed for $\mathrm{DL}_{\mathrm{CO}}$ and $\mathrm{K}_{\mathrm{CO}}$ that were in average moderately reduced ( $54 \pm 23 \%$ pred. and $55 \pm 22 \%$ pred., respectively). Indeed, all functional respiratory parameters (as \%pred.) were significantly worse in the FL subgroup than the NFL subgroup in these COPD patients. Notably, indices of pulmonary hyperinflation such as IC (\% pred.) and IC/TLC ratio (\%) and $\mathrm{DL}_{\mathrm{CO}}$ and $\mathrm{K}_{\mathrm{CO}}$ were markedly lower ( $\mathrm{p}$ $<0.05$ ) in the FL subgroup as compared to the NFL subgroup (Table 2).

Main data obtained by FOT are reported in Table 2, showing an increase in low-frequency respiratory system resistance (R,rs5) $(\mathrm{p}<0.05)$ and R,rs5-R,rs19 difference from NFL to FL patients, indicating a greater small airways obstruction in the FL subgroup $(\mathrm{p}<0.01)$. Moreover, $\mathrm{X}$,

Table 1

Clinical data of historical controls and clinical data and clinical parameters of COPD patients, all and divided according to absence (NFL) or presence (FL) of tidal expiratory flow limitation at rest.

\begin{tabular}{lllll}
\hline & COPD & NFL & FL & CTRL \\
\hline Subjects (n) & 19 & 12 & 7 & 20 \\
Clincal data & & & & $62 \pm 12$ \\
Age (y) & $66 \pm 9$ & $65 \pm 11$ & $67 \pm 5$ & $8 / 12$ \\
Sex (M/F) & $14 / 5$ & $8 / 4$ & $6 / 1$ & $172 \pm 9$ \\
Height (cm) & $169 \pm 8$ & $168 \pm 8$ & $171 \pm 6$ & $81 \pm 16$ \\
Weight (Kg) & $73 \pm 11$ & $73 \pm 12$ & $72 \pm 9$ & 27.4 \\
BMI & 25.0 & 25.7 & 24.5 & \\
Pack/Years & $51 \pm 23$ & $45 \pm 21$ & $60 \pm 25$ & \\
Smoke history (S/ExS) & $7 / 10$ & $6 / 4$ & $1 / 6$ & \\
mMRC & $2.1 \pm 1.3$ & $1.3 \pm 0.8$ & $3.2 \pm 0.8 * * *$ & \\
BODE & $4.4 \pm 3.2$ & $2.3 \pm 1.4$ & $8.0 \pm 1.9 * * *$ & \\
Clinical parameters & & & & \\
pH & $7.43 \pm 0.02$ & $7.44 \pm 0.01$ & $7.42 \pm 0.03$ & \\
PaCO2 (mmHg) & $40 \pm 3$ & $39 \pm 2$ & $43 \pm 3 * *$ & \\
PaO2 (mmHg) & $74 \pm 9$ & $78 \pm 8$ & $66 \pm 7 * *$ & \\
HCO3 (mMol/L) & $27 \pm 2$ & $26 \pm 1$ & $27 \pm 2$ & \\
$\Delta$ P(A-a)O2 & $26 \pm 8$ & $23 \pm 8$ & $31 \pm 5 *$ & \\
Hb (g/dL) & $14.8 \pm 1.6$ & $15.2 \pm 1.1$ & $14.1 \pm 2.1$ & \\
Lactate (mMol/L) & $0.9 \pm 0.4$ & $0.9 \pm 0.2$ & $1.1 \pm 0.6$ & \\
hs-CRP (mg/L) & $1.59 \pm 0.93$ & $1.23 \pm 0.76$ & $2.21 \pm 0.91$ & \\
Therapies (n) & & & & \\
LABA & 19 & 12 & 7 & \\
LAMA & 12 & 5 & 7 & \\
ICS & 8 & 2 & 6 & \\
OT (N/EF) & $4 / 4$ & 0 & & \\
\hline Data are mean & & & & \\
\hline
\end{tabular}

Data are mean \pm standard deviation.

$\mathrm{S}=$ current smokers; ExS $=$ ex-smokers.

hs-CRP $=$ high sensitivity C-Reactive Protein. OT = Oxygen therapy: $\mathrm{N}$ during night, EF during effort.

$*=\mathrm{p}<0.05 ; * *=\mathrm{p}<0.01 ; * * *=\mathrm{p}<0.001$ between NLF and FL groups.
Table 2

Respiratory functional parameters of COPD patients, all and divided according to absence (NFL) or presence (FL) of tidal expiratory flow limitation at rest.

\begin{tabular}{|c|c|c|c|}
\hline COPD & ALL & NFL & FL \\
\hline Subjects (n) & 19 & 12 & 7 \\
\hline SVC (L) & $3.70 \pm 0.9$ & $3.88 \pm 0.8$ & $3.39 \pm 0.9$ \\
\hline SVC (\% pred.) & $102.5 \pm 17.1$ & $110.8 \pm 12.1$ & $88.3 \pm 15.4 * *$ \\
\hline IC (L) & $2.43 \pm 0.6$ & $2.64 \pm 0.6$ & $2.08 \pm 0.6$ \\
\hline IC (\% pred.) & $87.5 \pm 19.0$ & $96.4 \pm 13.0$ & $72.5 \pm 17.2 * *$ \\
\hline FVC (L) & $3.20 \pm 0.8$ & $3.47 \pm 0.8$ & $2.74 \pm 0.7$ \\
\hline FVC (\% pred.) & $92.6 \pm 19.3$ & $103.2 \pm 14.5$ & $74.6 \pm 11.4 * * *$ \\
\hline $\mathrm{FEV}_{1}(\mathrm{~L})$ & $1.39 \pm 0.6$ & $1.68 \pm 0.5$ & $0.90 \pm 0.4 * *$ \\
\hline $\mathrm{FEV}_{1}$ (\% pred.) & $51.4 \pm 20.2$ & $62.7 \pm 12.7$ & $32.0 \pm 15.2 * * *$ \\
\hline $\mathrm{FEV}_{1} / \mathrm{SVC}$ (\% pred.) & $49.6 \pm 16.7$ & $57.5 \pm 12.8$ & $36.1 \pm 14.1 * *$ \\
\hline $\mathrm{PEF}(\mathrm{L} / \mathrm{s})$ & $3.92 \pm 1.6$ & $4.72 \pm 1.2$ & $2.55 \pm 1.1 * *$ \\
\hline PEF (\% pred.) & $54.3 \pm 21.6$ & $66.3 \pm 14.6$ & $33.9 \pm 15.5 * * *$ \\
\hline FEF $25-75(\%)$ & $0.50 \pm 0.3$ & $0.61 \pm 0.3$ & $0.32 \pm 0.1 *$ \\
\hline FEF $25-75(\%)$ & $16.6 \pm 8.4$ & $20.1 \pm 8.2$ & $10.6 \pm 4.3 *$ \\
\hline $\mathrm{DL}_{\mathrm{CO}}\left(\mathrm{ml} / \mathrm{min}^{*} \mathrm{mmHg}\right)$ & $13.45 \pm 6.3$ & $16.10 \pm 5.9$ & $8.91 \pm 4.1 *$ \\
\hline $\mathrm{DL}_{\mathrm{CO}}(\%$ pred.) & $54.1 \pm 22.7$ & $64.6 \pm 18.3$ & $36.0 \pm 18.3 * *$ \\
\hline $\mathrm{K}_{\mathrm{CO}}(\mathrm{ml} / \mathrm{min} * \mathrm{mmHg}) / \mathrm{L}$ & $2.42 \pm 1.24$ & $2.93 \pm 1.21$ & $1.56 \pm 0.72 *$ \\
\hline $\mathrm{K}_{\mathrm{CO}}(\%$ pred.) & $54.7 \pm 21.9$ & $63.6 \pm 18.9$ & $39.4 \pm 18.8 *$ \\
\hline $\mathrm{RV}(\mathrm{L})$ & $4.55 \pm 1.5$ & $3.85 \pm 1.1$ & $5.75 \pm 1.1 * *$ \\
\hline RV (\% pred.) & $194.3 \pm 59.2$ & $168.6 \pm 48.9$ & $238.3 \pm 50.7 * *$ \\
\hline FRC (L) & $5.36 \pm 1.3$ & $4.80 \pm 1.1$ & $6.32 \pm 1.1 * *$ \\
\hline FRC (\% pred.) & $160.6 \pm 33.7$ & $146.9 \pm 26.3$ & $184.1 \pm 33.3 *$ \\
\hline TLC (L) & $8.25 \pm 1.7$ & $7.72 \pm 1.6$ & $9.14 \pm 1.7$ \\
\hline TLC (\% pred.) & $133.0 \pm 18.6$ & $128.1 \pm 16.6$ & $141.3 \pm 20.2$ \\
\hline IC/TLC \% & $30.2 \pm 8.1$ & $34.5 \pm 4.9$ & $22.9 \pm 7.4 * * *$ \\
\hline R,rs $5 \mathrm{~Hz} \mathrm{cmH} 2 \mathrm{O} /(\mathrm{L} / \mathrm{s})$ & $4.16 \pm 1.0$ & $3.85 \pm 0.8$ & $4.69 \pm 1.2$ \\
\hline R,rs $5 \mathrm{~Hz}$ (\% pred.) & $156.9 \pm 49.7$ & $138.7 \pm 39.4$ & $188.2 \pm 52.3 *$ \\
\hline $\mathrm{R}, \mathrm{rs} 19 \mathrm{~Hz} \mathrm{cmH} 2 \mathrm{O} / \mathrm{L} / \mathrm{s})$ & $3.13 \pm 0.7$ & $3.12 \pm 0.5$ & $3.13 \pm 1.0$ \\
\hline R,rs $19 \mathrm{~Hz}$ (\% pred.) & $121.6 \pm 33.8$ & $117.4 \pm 27.7$ & $128.7 \pm 43.9$ \\
\hline R,rs 5-19 cmH2O/(L/s) & $0.92 \pm 0.6$ & $0.66 \pm 0.4$ & $1.39 \pm 0.6 * *$ \\
\hline $\mathrm{X}, \mathrm{rs} 5 \mathrm{~Hz} \mathrm{cmH} 2 \mathrm{O} /(\mathrm{L} / \mathrm{s})$ & $-3.03 \pm 2.67$ & $-1.52 \pm 0.48$ & $-5.61 \pm 2.93 * * *$ \\
\hline X,rs $5 \mathrm{~Hz}$ (\% pred.) & $287.0 \pm 286.2$ & $129.3 \pm 39.5$ & $537.5 \pm 328.4 * * *$ \\
\hline
\end{tabular}

Data are mean \pm standard deviation.

$\mathrm{PEF}=$ Peak Expiratory Flow; FEF 25-75\% = Forced Expiratory Flows between 25 and $75 \%$ of FVC.

$\mathrm{R}, \mathrm{rs}=$ Resistance of respiratory system; X,rs = Reactance of respiratory system. $*=\mathrm{p}<0.05 ; * *=\mathrm{p}<0.01 ; * * *=\mathrm{p}<0.001$ between NLF and FL groups.

rs5 was more negative in FL than in NFL patients, suggesting a deeper impairment of more peripheral, intra-acinar bronchioli in the FL subgroup ( $\mathrm{p}<0.001$ ). By definition, $\Delta \mathrm{X}$, rs5 was higher than $2.8 \mathrm{cmH}_{2} \mathrm{O} / \mathrm{L} / \mathrm{s}$ in the FL subgroup.

The frequency-domain analysis of supine HRV showed significantly higher LF and LF/HF ratio values in all COPD patients vs. Controls (always $\mathrm{p}<0.01$ ). Among different subgroups of COPD patients, NFL had $\mathrm{LF}$ and LF/HF ratio values similar to those of Controls and much lower than those observed in the FL subgroup ( $p<0.01$ and $\mathrm{p}<0.001$,

Table 3

Parameters obtained by frequency domain analysis of Controls and COPD patients (all and divided according to absence (NFL) or presence (FL) of tidal expiratory flow limitation at rest.

\begin{tabular}{lllll}
\hline & CTRL & COPD & NFL & FL \\
\hline Subjects (n) & 20 & 19 & 12 & 7 \\
HR sup & $69 \pm 11$ & $67.3 \pm 9.9$ & $64 \pm 8$ & $73 \pm 11^{*}$ \\
HR ortho & $77 \pm 10$ & $78.3 \pm 11.1$ & $77 \pm 10$ & $81 \pm 13$ \\
LF sup & $24.1 \pm 11.4$ & $40.9 \pm 20.1 \S$ & $31.4 \pm 17.8$ & $57.3 \pm 11.8 * *$ \\
HF sup & $40.0 \pm 16.0$ & $31.7 \pm 16.9$ & $36.8 \pm 19.1$ & $22.9 \pm 6.4$ \\
LF ortho & $52.5 \pm 22.9$ & $50.4 \pm 14.3$ & $50.7 \pm 15.0$ & $50.0 \pm 14.5$ \\
HF ortho & $17.1 \pm 9.3$ & $19.8 \pm 8.2$ & $20.1 \pm 8.6$ & $19.4 \pm 8.2$ \\
LF/HF sup & $0.7 \pm 0.4$ & $1.6 \pm 1.1 \S$ & $1.1 \pm 0.8$ & $2.6 \pm 0.7 * * *$ \\
LF/HF ortho & $3.5 \pm 1.6$ & $2.8 \pm 0.9$ & $2.8 \pm 1.0$ & $2.8 \pm 0.9$ \\
\hline
\end{tabular}

Data are mean \pm standard deviation. See text for abbreviations.

$\mathrm{HR}=$ heart rate; $\mathrm{LF}=$ power low frequency band; $\mathrm{HF}=$ power high frequency band; $\mathrm{LF} / \mathrm{HF}=$ ratio low/high frequency band power.

sup $=$ supine position; ortho $=$ standing position.

$\S=\mathrm{p}<0.05$ between Controls (CTRL) and COPD patients.

$* *=\mathrm{p}<0.01 ;{ }^{* * *}=\mathrm{p}<0.001$ between NFL and FL groups. 
respectively) (Table 3) and (Fig.1).

The LF/HF ratio was inversely related to $\mathrm{FEV}_{1}$ (\%pred.) $\left(\mathrm{r}^{2}=0.56, \mathrm{p}\right.$ $<0.001), \mathrm{FEV}_{1}$ /slow vital capacity (SVC) ratio (\%pred.) $\left(\mathrm{r}^{2}=0.53, \mathrm{p}<\right.$ $0.001)$ and $\mathrm{DL}_{\mathrm{CO}}\left(\%\right.$ pred.) $\left(\mathrm{r}^{2}=0.40, \mathrm{p}<0.01\right)$ and $\mathrm{K}_{\mathrm{CO}}\left(\%\right.$ pred.) $\left(\mathrm{r}^{2}=\right.$ $0.24, \mathrm{p}<0.05)$ and directly related to RV (\% pred.) and FRC (\% pred.) (Fig. 2 ab-cd, e-f).

Interestingly, LF/HF ratio was inversely related to IC/TLC ratio (\%) $\left(r^{2}=0.29, p<0.02\right)$ and albeit not significantly $\left(r^{2}=0.23, p=0.07\right)$ to IC (\%pred.) in our series (Fig. 3a). Indeed, the patients with COPD and significant dynamic hyperinflation at rest (IC/TLC ratio (\%) $<25 \%$ ) had a LF/HF ratio significantly higher than patients with COPD without dynamic hyperinflation at rest (IC/TLC ratio (\%) > $25 \%$ ) (Fig. 3b).

Three NFL patients could not perform the ISWT correctly, two because of back-pain and one because of an intercurrent leg muscle pain and were discarded for the analysis.

Distance walked during ISWT was significantly lower in the FL subgroup as compared with the NFL subgroup $(\mathrm{p}<0.01)$ with greater oxy-hemoglobin desaturation $(\mathrm{p}<0.01$ ) at the end of the exercise (Table 4). Moreover, $\triangle \mathrm{HRR} \mathrm{t}^{\prime}$ ' was markedly lower in the FL subgroup than that observed in the NFL subgroup $(\mathrm{p}<0.001)$ and always lesser than 14 beats/min, suggesting an abnormal autonomic cardiac activity in FL patients (Fig. 4a). In addition, $\Delta$ HRR $\mathrm{t}^{\prime}$ had a significant inverse relationship with $\mathrm{LH} / \mathrm{HF}$ ratio $\left(\mathrm{r}^{2}=0.42, \mathrm{p}<0.01\right)$ (Fig. 4) and a significant direct relationship with IC/TLC ratio $(\%)\left(\mathrm{r}^{2}=0.28, \mathrm{p}<0.05\right)$ and IC (\% pred.) $\left(r^{2}=0.37, \mathrm{p}<0.02\right)$ (Fig. $4 \mathrm{c}$ and $\mathrm{d}$ ).

Plasma levels of hs-CRP resulted positively related to LF/HF ratio $\left(\mathrm{r}^{2}\right.$ $=0.26, \mathrm{p}<0.05$ ) (Fig. 5a), but not inversely related to $\Delta$ HRR $\mathrm{t}^{\prime}\left(\mathrm{r}^{2}=\right.$ 0.19 , not significant). The hs-CRP plasma levels, although not significantly related to both IC (\%pred.) $\left(\mathrm{r}^{2}=0.04\right)$ and IC/TLC ratio $(\%)\left(\mathrm{r}^{2}=\right.$ $0.07)$, were higher in the FL subgroup as compared to the NFL subgroup (p $<0.05$ ) (Fig. 5b).

\section{Discussion}

The findings of the study confirm that in patients with stable COPD, there is an autonomic nervous system activity imbalance with cardiac sympathetic predominance, as shown by higher LF/HF ratio, higher LF and lower HF power in the frequency domain supine HRV analysis, which is significantly related to the severity of airflow obstruction, the occurrence of pulmonary hyperinflation, and prevalence of the emphysematous disease. Also, a relationship between autonomic dysfunction and systemic inflammation, as assessed by increased hs-CRP plasma levels, has been observed again, suggesting an association between these phenomena whose significance and direction remain to be established.

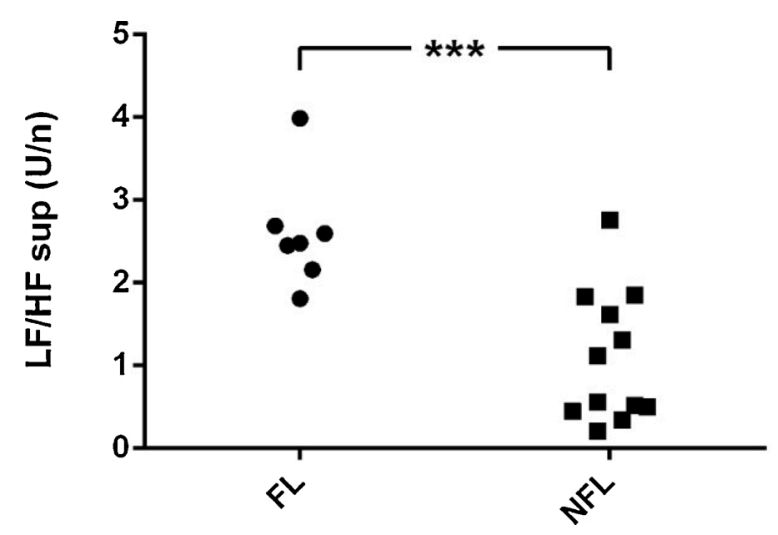

Fig. 1. Comparison of supine LF/HF ratio in patients with COPD divided according to the presence of tidal expiratory flow limitation at rest. LF/HF ratio is significantly higher in FL than NFL patients, showing that the cardiac autonomic imbalance is strongly linked to the presence of resting tidal FL. *** $\mathrm{p}<$ 0.001 . The horizontal lines represent the mean values.
However, the novelty of the study is that autonomic dysfunction and low-grade systemic inflammation more than with COPD per se are associated with resting tidal FL in patients with COPD. This emerges clearly from our data showing that either LF/HF ratio increase and $\triangle$ HRR $\mathrm{t}^{\prime}$ ' decrease, both markers of autonomic system nervous imbalance toward predominant cardiac sympathetic activity, were significantly greater in FL as compared with NFL patients. Concurrently, significantly higher plasma levels of hs-CRP, an established IL-6-related biomarker of systemic inflammation when persistently elevated, were found in FL patients versus NFL patients.

The association between cardiac sympathetic dominance and systemic inflammation in COPD has been hypothesized in the past and demonstrated in different cohorts of patients with COPD in two previous studies (Chhabra et al., 2015; Corbo et al., 2013). In the latter, a role of lung function impairment and notably the presence of pulmonary hyperinflation, assessed by the IC/TLC ratio (\%), was also postulated as a potential contributor to this relationship (Corbo et al., 2013).

Since resting tidal FL promotes the occurrence of dynamic pulmonary hyperinflation, very often at rest and invariably during exercise and sleep, this mechanical condition and its negative consequences (increased load on inspiratory muscles, greater negative intrathoracic pressure swings, altered venous return to the right heart and left heart diastolic dysfunction) might be a pathophysiological link between autonomic dysfunction and systemic inflammation (O'Donnell et al., 2001).

In this respect, despite both $\mathrm{LF} / \mathrm{HF}$ ratio and $\Delta \mathrm{HRR} \mathrm{t} 1^{\prime}$ were related to several functional parameters reflecting pulmonary hyperinflation such as FRC (\%pred.), IC (\%pred.), and IC/TLC ratio (\%), in contrast with what previously observed (Gatta et al., 2011), we did not find a significant relation between hs-CRP plasma levels and indices of pulmonary hyperinflation, such IC (\%pred.) and IC/TLC ratio (\%). This controversial aspect needs to be further clarified, probably exploring larger cohorts of COPD patients.

These findings underline several aspects of COPD's heterogeneity that cannot be explained by the severity of airflow obstruction alone. Resting pulmonary hyperinflation appears tightly related to the development of autonomic derangement at least of cardiac activity in these patients, and the presence of tidal FL is crucial for the occurrence of the dynamic component of pulmonary hyperinflation at rest, during the effort, and sleep. Tidal FL, which represents a remarkably disadvantageous lung mechanical constraint, is rarely searched by pneumologists because of its inherent difficulties (Tantucci, 2013), but progressive FOT implementation in respiratory function labs can make it easier to detect (Dellacà et al., 2004).

Both $\mathrm{DL}_{\mathrm{CO}}$ and $\mathrm{K}_{\mathrm{CO}}$ appear related to autonomic dysfunction, as assessed by either LF/HF ratio or $\triangle \mathrm{HRR}$, suggesting that the prevalence of the emphysematous component of COPD may play a role in the sympathetic and parasympathetic cardiac activity modulation. The negative influence of advanced pulmonary emphysema on heart size and heart dysfunction observed in COPD (Jorgensen et al., 2003), or the earlier development of tidal FL in emphysematous patients (Chiari et al., 2014), can reasonably sustain this possibility.

Furthermore, the fairly good relationship between $\Delta \mathrm{HRR} \mathrm{t} \mathrm{1}^{\prime}$ and LF/ HF ratio in establishing the presence of cardiac autonomic dysfunction and the difficulty of performing HRV analysis routinely in COPD patients makes $\triangle$ HRR $\mathrm{t}^{\prime}$ a simple, very attractive parameter for assessing this potentially dangerous condition.

Altogether, we have useful data to suspect of the presence of a relevant autonomic imbalance in patients with COPD presenting tidal FL, pulmonary hyperinflation, and prevalence of pulmonary emphysema, and FOT and pulmonary function tests for assessing IC and/or IC/ TLC ratio $\%$ and $\mathrm{DL}_{\mathrm{CO}}$ and $\mathrm{K}_{\mathrm{CO}}$ may be easy tools to measure it.

In this context, the use of selective beta-blocking drugs appears welcome in patients with COPD with the characteristics mentioned above, thus supporting the therapeutic utility of these drugs in terms of morbidity and cardiac and all-cause mortality in COPD, widely 

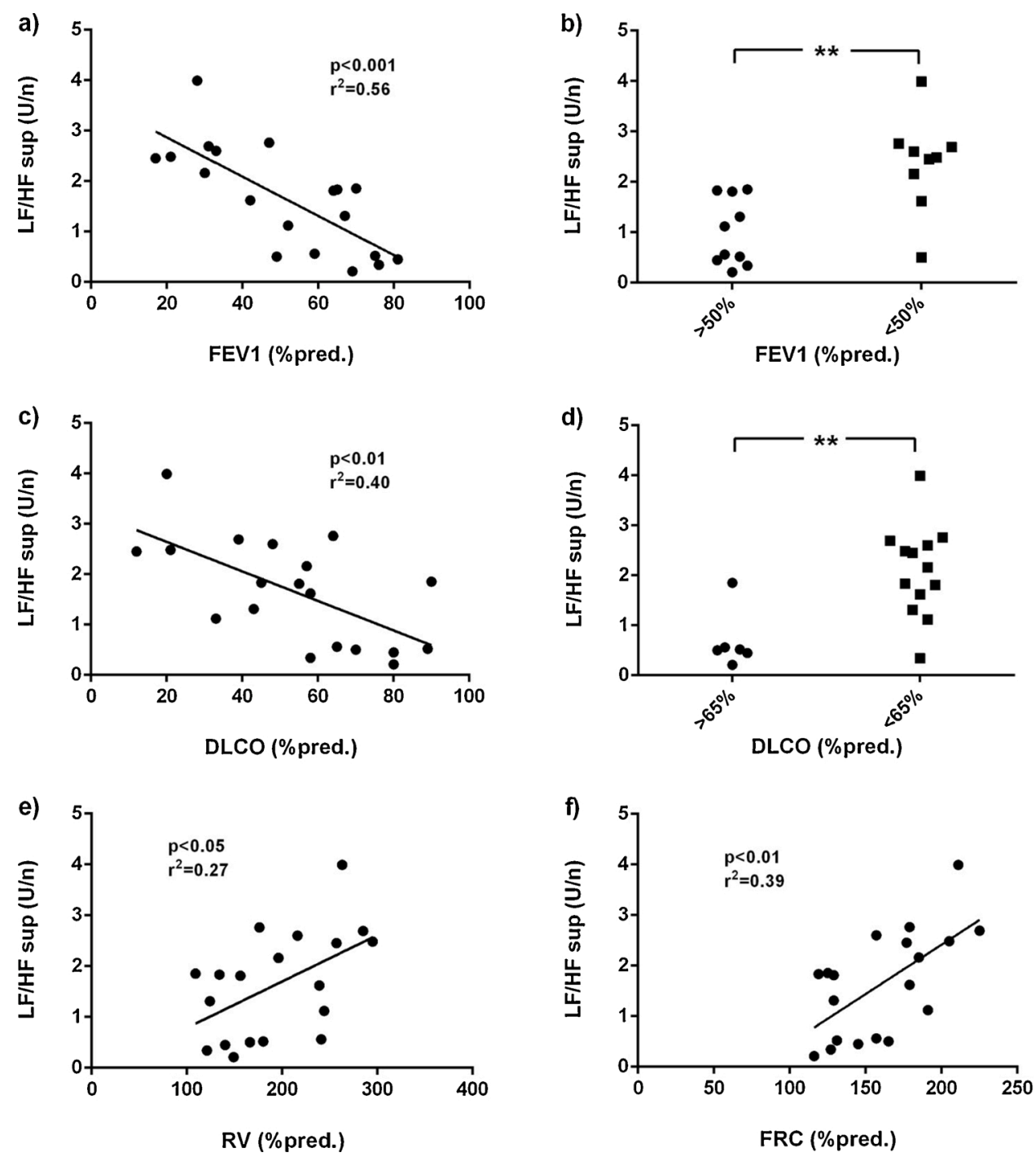

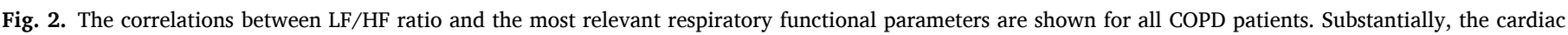

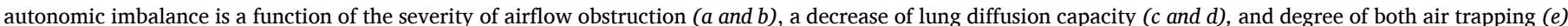
and pulmonary hyperinflation $(f)$. ${ }^{* *} \mathrm{p}<0.01$.
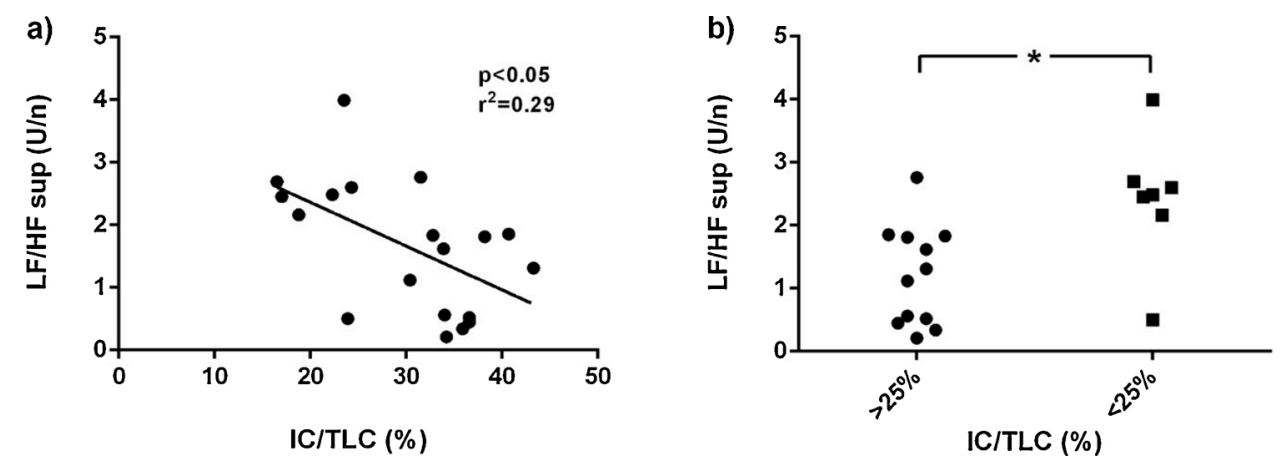

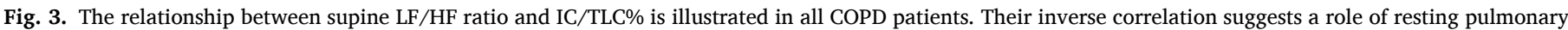

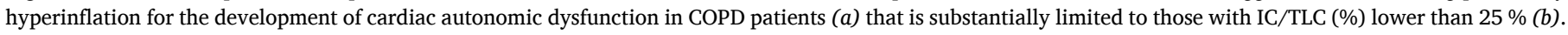
* $\mathrm{p}<0.05$.

documented in previous epidemiological studies (Rutten et al., 2010; Short et al., 2011; Beta-blocker use and COPD mortality: a systematic review and meta-analysis, 2012).

Recently, however, a prospective, randomized, placebo-controlled trial, looking at the effect of metoprolol (extended-release) on time until the first exacerbation in moderate-to-severe COPD patients who should not receive beta-blocker drugs for other medical reasons, did not find any significant difference as compared to placebo about freedom from COPD exacerbation, showing, in turn, a higher risk of hospitalization for severe COPD exacerbations in the metoprolol group 
Table 4

Data obtained during Incremental Shuttle Walking Test (ISWT) in COPD patients, all and divided according to absence (NFL) or presence (FL) of tidal expiratory flow limitation at rest.

\begin{tabular}{llll}
\hline COPD & ALL & NFL & FL \\
\hline Subjects (n) & 16 & 9 & 7 \\
ISWT (m) & $357 \pm 166$ & $430 \pm 164$ & $231 \pm 69 * *$ \\
ISWT (\% pred.) & $50 \pm 17$ & $60 \pm 11$ & $31 \pm 9 * * *$ \\
SpO2 pre \% & $96 \pm 2$ & $96 \pm 1$ & $95 \pm 3$ \\
SpO2 \% min & $88 \pm 7$ & $91 \pm 5$ & $82 \pm 8 * *$ \\
$\Delta$ SpO2 \% & $-7 \pm 6$ & $-5 \pm 4$ & $-12 \pm 6 * *$ \\
BORG pre & $0.3 \pm 0.8$ & $0.0 \pm 0.0$ & $0.8 \pm 1.2 *$ \\
BORG post & $5.6 \pm 2.0$ & $4.6 \pm 1.6$ & $7.3 \pm 1.5 * *$ \\
SBP pre (mmHg) & $135 \pm 16$ & $132 \pm 14$ & $139 \pm 18$ \\
SBP post (mmHg) & $174 \pm 25$ & $174 \pm 26$ & $175 \pm 25$ \\
DBP pre (mmHg) & $80 \pm 11$ & $78 \pm 13$ & $83 \pm 8$ \\
DBP post (mmHg) & $92 \pm 14$ & $89 \pm 14$ & $96 \pm 13$ \\
HR pre (beat/min) & $71 \pm 12$ & $68 \pm 8$ & $77 \pm 15$ \\
HR max (beat/min) & $111 \pm 14$ & $112 \pm 15$ & $111 \pm 11$ \\
HRR t 1' (beat/min) & $99 \pm 14$ & $95 \pm 16$ & $103 \pm 10$ \\
$\Delta$ HRR t 1' (beat/min) & $-14 \pm 7$ & $-19 \pm 6$ & $-7 \pm 2 * * *$ \\
HRR t 2' (beat/min) & $85 \pm 11$ & $81 \pm 12$ & $89 \pm 9$ \\
HRR t 3' (beat/min) & $76 \pm 11$ & $72 \pm 10$ & $81 \pm 9$ \\
\hline
\end{tabular}

Data are mean \pm standard deviation.

ISWT (incremental shuttle walking test);

$\mathrm{SpO} 2$ pre = baseline oxyhemoglobin pulse-oximeter saturation;

SpO2 $\min =$ minimum oxyhemoglobin pulse-oximeter saturation during ISWT;

$\Delta \mathrm{SpO} 2=$ difference between $\mathrm{SpO} 2$ pre and $\mathrm{SpO} 2 \mathrm{~min}$;

$\mathrm{SBP}$ and DBP = systolic and diastolic blood pressure;

HR pre = baseline heart rate; HR max = maximum heart rate during ISWT;

$\mathrm{HRR}=$ Heart Rate Recovery after $1 \mathrm{~min}\left(\mathrm{t} 1^{\prime}\right), 2 \mathrm{~min}\left(\mathrm{t} 2^{\prime}\right)$ and $3 \mathrm{~min}\left(\mathrm{t} 3^{\prime}\right)$ from. stopping exercise. $\triangle \mathrm{HRR}=$ Change of Heart Rate Recovery after $1 \mathrm{~min}\left(\mathrm{t} 1^{\prime}\right)$.

$*=\mathrm{p}<0.05 ;{ }^{* *}=\mathrm{p}<0.01 ; * * *=\mathrm{p}<0.001$ between NFL and FL groups.
(Dransfield et al., 2019). Apart from the questionable choice of the primary end-point (i.e., the prevention of the COPD-related exacerbations), some limitations of this study could explain these findings, such as the cohort of the COPD patients who were characterized by a high prevalence of supplemental oxygen use and previous hospitalization for COPD (Ekstrom et al., 2013) and the cardio-selective beta-blocker selected for the trial (Su et al., 2016).

Ultimately, in patients with COPD, the association between FL with related pulmonary hyperinflation and autonomic dysfunction and systemic inflammation seems to come out, although not causally clarified, adding relevance to the interplay between functional impairment and biological consequences as key mechanism to the development of comorbidities in COPD.

\subsection{Study limitations}

The small number of patients with COPD enrolled can certainly be a limitation concerning this study's findings. However, looking for a broad range of COPD severity, many patients were excluded because of the relevant comorbidities and associated drugs they assumed. Also, we did not include COPD patients with a history of recent frequent exacerbations. Moreover, we tried to be very accurate in collecting each data set, and given the complexity of the protocol, some other patients did not complete carefully all the tests and were discarded from the analysis. Thus, if the possibility does exist that our findings were obtained by chance (type I error), on the contrary, we are confident about our results and their significance, if anything, limited by a type II error.
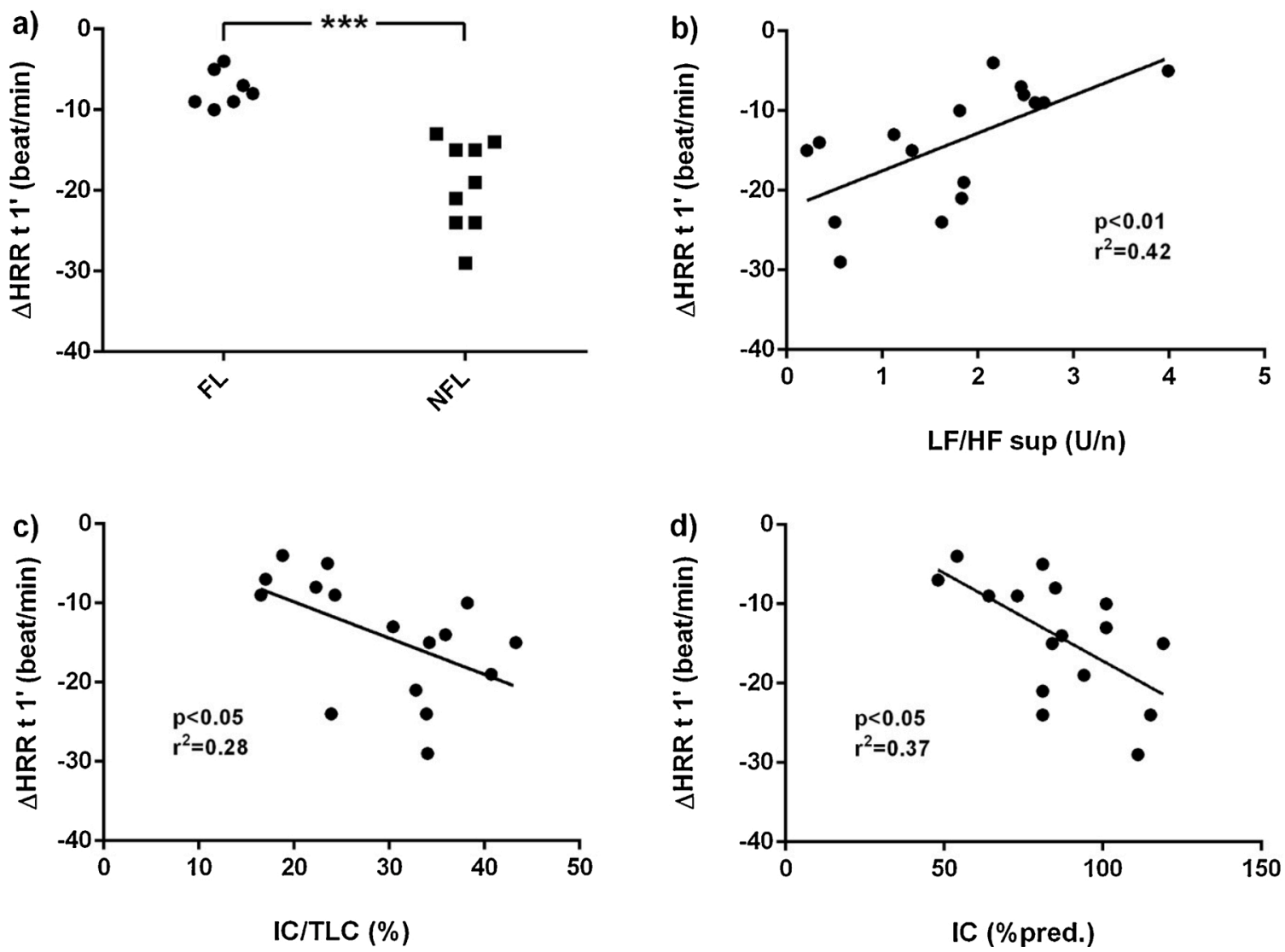

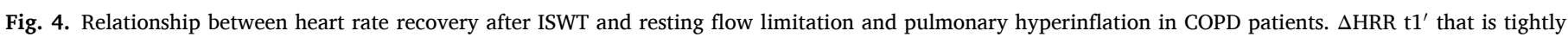

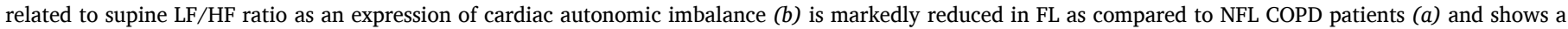
direct correlation with both IC/TLC\% and IC (\%pred.) ( $c$ and d). ${ }^{* * *} \mathrm{p}<0.001$. 

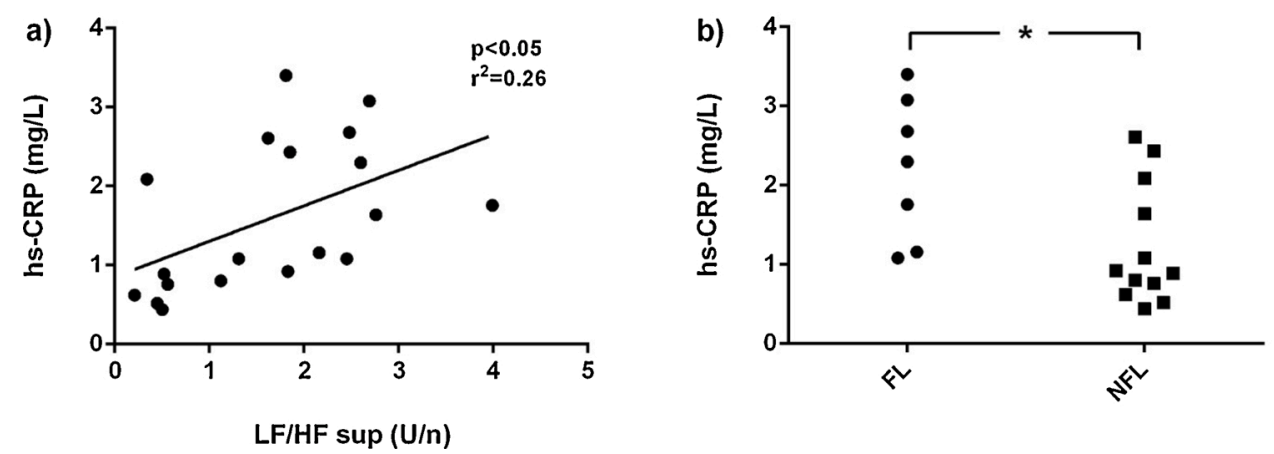

Fig. 5. Relationship between systemic inflammation and autonomic cardiac imbalance and its presence in FL and NFL patients. The hs-CRP plasma levels are directly related to the LF/HF ratio (a) and are significantly augmented in FL compared to NFL COPD patients $(b) .{ }^{*} \mathrm{p}<0.05$.

\section{Conclusions}

In stable COPD patients, greater airflow obstruction, lower lung diffusing capacity, and larger lung volumes were associated with autonomic dysfunction, characterized by abnormal sympathetic cardiac modulation. LF/HF ratio was significantly related to both hs-CRP plasma levels and indices of dynamic pulmonary hyperinflation, such as the IC/ TLC ratio (\%) and FRC (\% pred.). The presence of resting tidal FL, favoring dynamic hyperinflation, was a strong driver of either abnormal autonomic cardiac activity, as assessed by an elevated LF/HF ratio and low $\Delta$ HRR $\mathrm{t}^{\prime}$, or systemic inflammation, as assessed by high plasma levels of hs-CRP, suggesting a significant interplay among dynamic pulmonary hyperinflation, autonomic dysfunction and low-grade systemic inflammation in steadily flow-limited COPD patients.

\section{Acknowledgements}

Study design: CT. Data collection: DB, FS, RM, LP. Data analysis: LP and CT. Interpretation of results: all authors. Initial draft: CT. Review of the manuscript for intellectual content: all authors.

The Authors acknowledge E. Boni M.D. for his skilled support in the HRV analysis and Mr. M. Guerini, Lab. technician, for his invaluable technical support.

\section{References}

Beta-blocker use and COPD mortality: a systematic review and meta-analysis, 2012. Beta-blocker use and COPD mortality: a systematic review and meta-analysis. BMC Pulm. Med. 12, 48-54.

Borovikova, L.V., Ivanova, S., Zhang, M., et al., 2000. Vagal nerve stimulation attenuates the systemic inflammatory response to endotoxin. Nature 405, 458-462.

Chhabra, S.K., Gupta, M., Ramaswamy, S., Dash, D.J., Bansal, V., Deepak, K.K., 2015. Cardiac sympathetic dominance and systemic inflammation in COPD. COPD 12, $552-559$.

Chiari, S., Bassini, S., Braghini, A., Corda, L., Boni, E., Tantucci, C., 2014. Tidal expiratory flow limitation at rest as functional marker of pulmonary emphysema in moderate-to.-Severe chronic obstructive pulmonary disease. COPD 11, 33-38.

Corbo, G.M., Inchingolo, R., Sgueglia, G.A., Lanza, G., Valente, S., 2013. C-reactive protein, lung hyperinflation and heart rate variability in Chronic Obstructive Pulmonary Disease - a pilot study. COPD 200-207.

Dellacà, R.L., Santus, P., Aliverti, A., et al., 2004. Detection of expiratory flow limitation using the forced oscillation technique. Eur. Respir. J. 23, 232-240.
Dellacà, R.L., Duffy, N., Pompilio, P.P., et al., 2007. Expiratory flow limitation detected by forced oscillation and negative expiratory pressure. Eur. Respir. J. 29, 363-374. Dransfield, M.T., Voelker, H., Bhatt, S.P., et al., 2019. Metoprolol for the prevention of acute exacerbations of COPD. New England J. Med. Surg. Collat. Branches Sci. 381, 2304-2314.

Ekstrom, M.P., Hermansson, A.B., Strom, K.E., 2013. Effects of cardiovascular drugs on mortality in severe chronic obstructive pulmonary disease. Am. J. Respir. Crit. Care Med. 187, 715-720.

Gatta, D., Aliprandi, G., Pini, L., Zanardini, A., Fredi, M., Tantucci, C., 2011. Dynamic pulmonary hyperinflation and low grade systemic inflammation in stable COPD patients. Eur. Rev. Med. Pharmacol. Sci. 15, 1068-1073.

Heindle, S., Lehnert, M., Crièe, C.P., 2001. Marked sympathetic activation in patients with chronic respiratory failure. Am. J. Respir. Crit. Care Med. 164, 597-601.

Jorgensen, K., Houltz, E., Westfel, U., Nilsson, F., Sherstein, E., Rcksten, S.E., 2003. Effects of lung volume reduction surgery on left ventricular diastolic filling and dimensions in patients with severe emphysema. Chest 124, 1863-1870.

Lacasse, M., Maltais, F., Poirier, P., et al., 2005. Post-exercise heart rate recovery and mortality in chronic obstructive pulmonary disease. Resp Med 99, 877-886.

Marz, P., Cheng Jr., -G., Gadiente, R.A., et al., 1998. Sympathetic neurons can produce and respond to interleukin 6. Proc Natl Acad Sci 95, 3251-3256.

Miller, M.R., Crapo, R., Hankinson, J., et al., 2005. General considerations for lung function testing. Eur. Respir. J. 26, 153-1611.

Mohamed, J., Meeus, M., Derom, E., Da Silva, H., Calders, P., 2015. Evidence for autonomic function and its influencing factors in subjects with COPD: a systematic review. Respir. Care 12, 1841-1851.

O’Donnell, D.E., Revil, S.M., Webb, K.A., 2001. Dynamic hyperinflation and exercise intolerance in chronic obstructive pulmonary disease. Am. J. Respir. Crit. Care Med. 164, 770-777.

Quanjer, P.H., Tammeling, G.J., Cotes, J.E., Pedersen, O.F., Peslin, R., Yernault, J.C., 1993. Lung volumes and forced ventilatory flows. Eur. Respir. J. 6 (Suppl 16), 5-40.

Rutten, F.H., Zuihtoff, N.P., Hak, E., Grobbee, D.E., Hoes, A.W., 2010. Beta-blockers may reduce mortality and risk of exacerbation in patients with chronic obstructive pulmonary disease. Arch. Intern. Med. 170, 880-887.

Short, P.M., Lipworth, S.I., Elder, D.H., Schembri, S., Lipworth, B.J., 2011. Effect of betablockers in treatment of chronic obstructive pulmonary disease: a retrospective cohort study. BMJ 342, d2549.

Su, V.Y., Chang, Y.S., Hu, Y.W., et al., 2016. Carvedilol, Bisoprolol, Metoprolol use in patients with coexistent heart failure and chronic obstructive pulmonary disease. Medicine 95, e2427.

Tantucci, C., 2013. Expiratory flow limitation: definition, mechanisms methods and significance. Pulm. Med., 749860

Task Force of European Society of Cardiology and the North American Society of Pacing and Electrophysiology, 1996. Heart rate variability. Standards of measurement, physiological interpretation and clinical use. Circulation 93, 1043-1065.

Tracey, K.J., 2002. The inflammatory reflex. Nature 420, 853-859.

Van Gestel, A.J.R., Steier, J., 2010. Autonomic dysfunction in patients with chronic obstructive pulmonary disease. J. Thorac. Dis. 2, 15-122.

Wang, H., Yu, M., Ochani, M., et al., 2003. Nicotine acetylcholine receptor alpha7 subunit is an essential regulator of inflammation. Nature 421, 384-388. 